\title{
Disease Watch
}

\section{SECRETS OF MDR-TB REVEALED}

A study reports that the high prevalence of multidrug-resistant (MDR) tuberculosis (TB) and extensively drug-resistant (XDR) TB in Samara, Russia, is caused not only by deficiencies in the TB control programme but also by an unusual combination of resistance and compensatory mutations that enables Mycobacterium tuberculosis strains to retain clinical resistance without losing fitness or transmissibility. Whole-genome sequencing of 1,000 patient isolates revealed that $48 \%$ of all isolates were MDR and $16 \%$ of these were XDR; however, compensatory mutations that ameliorate the fitness costs of resistance were widespread. For example, compensatory mutations in over 400 rifampicin-resistant isolates were identified, including a newly discovered intragenic mutation in rpoB (encoding RNA polymerase $\beta$ subunit). Furthermore, $65 \%$ of MDR isolates carried eis promoter mutations that cause kanamycin resistance, suggesting that extensive use of kanamycin might select for MDR-TB and enhance transmission.

ORIGINAL RESEARCH PAPER Casali, N. et al. Evolution and transmission of drug-resistant tuberculosis in a Russian population. Nature Genet. http://dx.doi. org/10.1038/ng.2878 (2014)

\section{ADAPTING TO A NASAL LIFESTYLE}

Using a combined metabolomics and transcriptomics approach, a new study reports that Staphylococcus aureus is highly dependent on de novo methionine biosynthesis to efficiently colonize the human nose. Accordingly, growth of S. aureus in a synthetic medium that mimicked the nasal environment was dependent on the methionine biosynthesis enzyme cysteine- $\gamma$-synthase (Metl), and metl mutants showed a decreased ability to colonize the noses of cotton rats. S. aureus colonization of the human nose is a major risk factor for invasive infections, but the authors report that a Metl inhibitor blocks bacterial growth, which suggests that this biosynthetic pathway could be a new antibacterial target.

ORIGINAL RESEARCH PAPER Krismer, B. et al. Nutrient limitation governs Staphylococcus aureus metabolism and niche adaptation in the human nose. PLoS Pathog. 10, e1003862 (2014)

\section{OUTBREAK NEWS}

H7N9. The incidence of human infections with the H7N9 avian influenza virus in China has declined since May 2013 owing to a number of interventions; however, so far in 2014, 100 infections and at least 19 deaths have been reported. The resurgence of the virus has been attributed to increased virus circulation during the winter months and to the rise in travel and live poultry trading ahead of the Chinese New Year. The Guardian/BBC News/WHO

Chikungunya virus. The mosquito-borne chikungunya virus is spreading rapidly in the Caribbean, which is the first time indigenous transmission of the virus has been documented in the Americas. Thus far, $>780$ confirmed and/or probable cases have been reported, and public health officials are urging for vector control strategies to be implemented to curb the outbreak. PAHO/WHO/ECDC 